\title{
A method for optimal fit of patient-specific fracture fixation plates
}

\begin{abstract}
Anatomically pre-contoured fracture fixation plates are a treatment option for bone fractures. A well-fitting plate can be used as a tool for anatomical reduction of the fractured bone. However, recent studies showed that some plates fit poorly for many patients due to considerable shape variations between bones of the same anatomical site. Therefore, the plates have to be manually fitted and deformed by surgeons to fit each patient optimally. The process is time-intensive and labor-intensive, and could lead to adverse clinical implications such as wound infection or plate failure. This paper proposes a new iterative method to simulate the patient-specific deformation of an optimally fitting plate for pre-operative planning purposes. We further demonstrate the validation of the method through a case study. The proposed method involves the integration of four commercially available software tools, Matlab, Rapidform2006, SolidWorks, and ANSYS, each performing specific tasks to obtain a plate shape that fits optimally for an individual tibia and is mechanically safe. A typical challenge when crossing multiple platforms is to ensure correct data transfer. We present an example of the implementation of the proposed method to demonstrate successful data transfer between the four platforms and the feasibility of the method.
\end{abstract}

Keyword: Patient-specific implant; Anatomical fit; Fit assessment; Fracture fixation; Orthopedic plate 